\title{
Photoneutron Flux Measurement via Neutron Activation Analysis in a Radiotherapy Bunker with an 18 MV Linear Accelerator
}

\author{
Yiğit Çeçen ${ }^{1,2, *}$, Tuğçe Gülümser ${ }^{1,2}$, Çağrl Yazgan $^{1,2}$, Haris Dapo $^{1},{\text { Mahmut } \text { Üstün }^{1} \text {,Ismail Boztosun }}^{1}$ \\ ${ }^{1}$ Akdeniz University, Nuclear Sciences Application and Research Center, 07070, Antalya, Turkey \\ ${ }^{2}$ Akdeniz University, School of Medicine, Department of Radiation Oncology, 07070, Antalya, Turkey
}

\begin{abstract}
In cancer treatment, high energy X-rays are used which are produced by linear accelerators (LINACs). If the energy of these beams is over $8 \mathrm{MeV}$, photonuclear reactions occur between the bremsstrahlung photons and the metallic parts of the LINAC. As a result of these interactions, neutrons are also produced as secondary radiation products $(\gamma, n)$ which are called photoneutrons. The study aims to map the photoneutron flux distribution within the LINAC bunker via neutron activation analysis (NAA) using indium-cadmium foils. Irradiations made at different gantry angles $\left(0^{\circ}, 90^{\circ}, 180^{\circ}\right.$ and $\left.270^{\circ}\right)$ with a total of 91 positions in the Philips SLI-25 linear accelerator treatment room and location-based distribution of thermal neutron flux was obtained. Gamma spectrum analysis was carried out with high purity germanium (HPGe) detector. Results of the analysis showed that the maximum neutron flux in the room occurred at just above of the LINAC head $\left(1.2 \times 10^{5}\right.$ neutrons $\left./ \mathrm{cm}^{2} . s\right)$ which is compatible with an americium-beryllium (Am-Be) neutron source. There was a $90 \%$ decrease of flux at the walls and at the start of the maze with respect to the maximum neutron flux. And, just in front of the LINAC door, inside the room, neutron flux was measured less than $1 \%$ of the maximum.
\end{abstract}

\section{Introduction}

Cancer is one of the most common health problem known and the number of cancer patients increase every year all over the world [1]. New methods for cancer treatment are developed with developing technology [2]. One of the methods developed for the treatment of cancer patients is the treatment by radiation, known as radiotherapy.

Radiotherapy centers use high energy X-rays produced by LINACs to treat patients. Electrons are accelerated into a tungsten target to produce bremsstrahlung photons [3]. These photons are produced by electrons at different energies ranging from 4 to 25 MeVs.

By design, there are high atomic number materials inside the accelerator head (such as target, smoothing filter, collimators). If the bremsstrahlung X-rays' energy exceeds the threshold energy of these materials, photonuclear reactions such as neutron break off may occur. These neutrons are referred to as photoneutrons. Produced neutrons may cause a nuclear reaction interacting with the surrounding material. These materials, which become active with $(n, \gamma)$ reactions, may release gamma radiation to the environment [4].
Resulting photoneutrons spread out easily from the accelerator head and scatter into the room. As a result of these neutrons, an additional dose occurs in the patient's body [5]. In addition to unwanted additional dose in the patient; due to high radiobiological effects of the neutrons, significant amount of side effects may develop for the patient [6]. These unwanted doses and radiobiological activity also increase the risk of secondary cancer [7]. Products resulting from the photoactivation event also threaten the health of the employees and the people who are inside or outside facility $[5,8,9]$. Electronic devices in the treatment room are also adversely affected by the production of photoneutrons $[9,10]$. All of the materials found in the LINAC room are capable of doing neutron absorption reactions [11].

Flux distribution of the photoneutrons in the treatment room depends on many variables. The room design hosting the accelerator (the room's geometry and consisting materials of the shielding), the type of accelerator (design and materials it contains), the accelerator's radiation beam type, the planned treatment area (wedge, multi-leaf collimator, etc.) and the resulting neutron spectrum are the factors that affect photoneutron distribution in the treatment room [5].

* Corresponding author: ycecen $@$ akdeniz.edu.tr 
To create a suitable radiation protection protocol for the device, deposited neutron dose by workers and patients should be calculated. To calculate the dose of the occurring neutrons, characteristics of neutrons in the therapy room (flux, distribution, etc.) must be known [12].

\section{Materials \& Methods}

To obtain the neutron distribution in the radiotherapy room, thermal neutron flux was measured in different locations. Thermal neutrons will generate more radiation damage in living tissue and in circuit materials due to high thermal absorption cross sections of water, carbon and silicon [13].

In this study, Philips SLI-25 linear accelerator was used as a photo-nuclear source. For the analysis AMATEK-ORTEC brand GMX-20195-P model n-type high purity germanium (HPGe) semi-conductor detector was used.

Indium and cadmium metal foils were used for neutron activation analysis (NAA), which both have high neutron absorption cross sections. These foils are activated as a result of neutron absorption. As shown in figure 2.1, indium cross section is high for thermal and epithermal energy regions [14]. Measurements with bare indium foils gave information about the neutrons in these energy ranges.

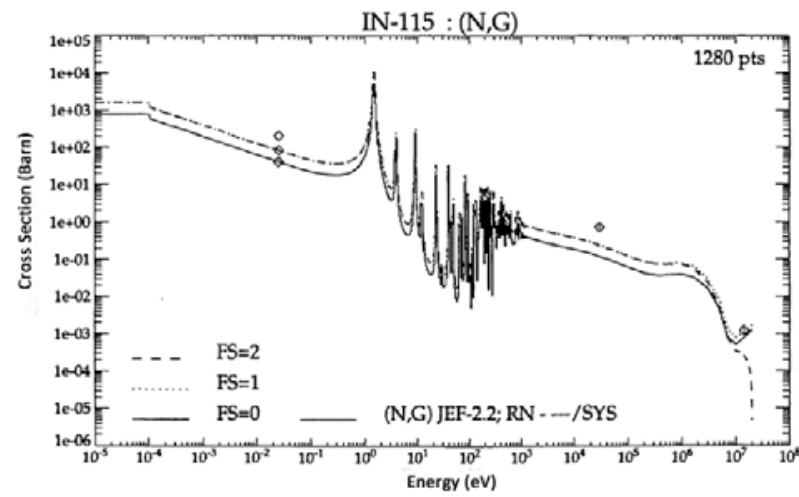

Figure 2.1. Indium-115 Neutron Absorption Cross Section [15]

The cadmium cross section is high for neutrons in thermal and high energy regions (Figure 2.2) [14]. For this reason, indium foils were covered with the cadmium foils and same experimental setup was prepared and measurements were taken. Thus, thermal neutrons were absorbed by cadmium before they reach the indium and only epithermal neutrons were absorbed by the indium foils.

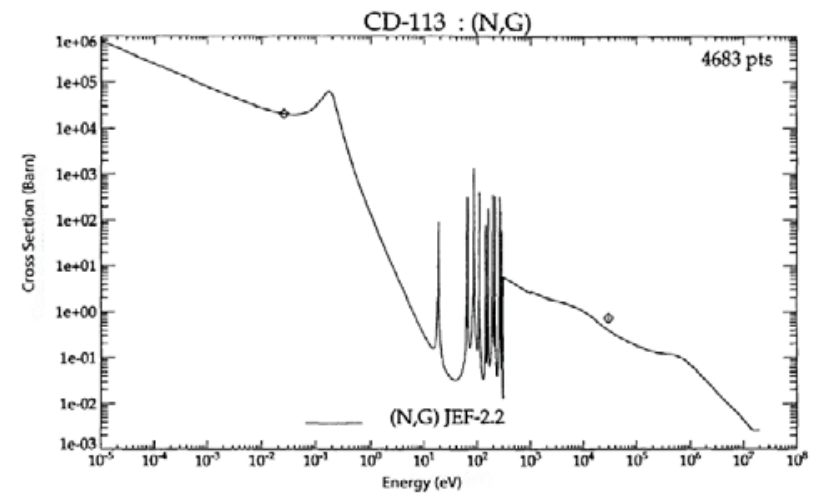

Figure 2.2. Cadmium-113 Neutron Absorption Cross Section [15]

To obtain the information for the thermal neutron flux, differences between the measured data must be studied between bare indium and cadmium covered indium foils.

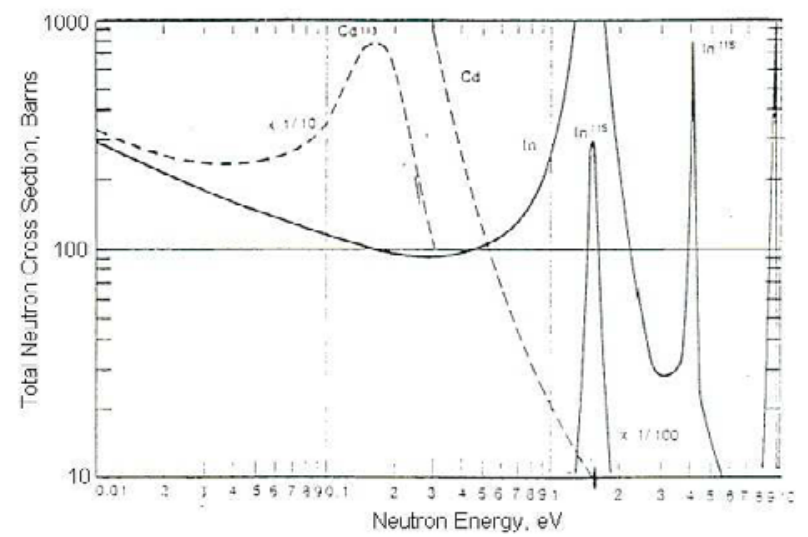

Figure 2.3. Total Neutron Cross Section Comparison of Cadmium-113 and Indium-115

$$
\begin{aligned}
& { }_{0}^{1} \mathrm{n}+{ }_{49}^{115} \mathrm{In} \rightarrow{ }_{49}^{\mathrm{T}_{\mathrm{V} / 2}=54.2 \mathrm{~min}} \mathrm{In} \stackrel{{ }_{\mathrm{T} / 2}=14 \mathrm{~s}}{\rightarrow} \\
& { }_{50}^{116} \mathrm{Sn} *+\beta^{-}+\bar{v} \stackrel{\mathrm{T}_{\mathrm{T} / 2}=14.10 \mathrm{~h}}{\rightarrow}{ }_{50}^{116} \mathrm{Sn}+\gamma
\end{aligned}
$$

As shown in eq. 2.1, ${ }^{115}$ In absorbs one neutron and then it becomes ${ }^{116} \mathrm{In}$ in 14 seconds. Half-life of ${ }^{116} \mathrm{In}$ is 54.2 minutes. ${ }^{116}$ In isotope at excited state emits $\beta^{-}$radiation because of neutron excess. After the decay, ${ }^{116}$ In becomes ${ }^{116} \mathrm{Sn}$ which has a half-life of 4.1 hours. During this process an anti-neutrino is emitted by ${ }^{116} \mathrm{In}$ for energy and momentum protection [16]. Experiments were carried out for different locations by resetting the activity of indium foils. Due to 4.1 hours half-life of ${ }^{116} \mathrm{Sn}$, two days of cooling period was planned between experiments which correspond to about ten half-lives.

For the analysis of photoneutron flux in the radiotherapy room, $18 \mathrm{MeV}$ bremsstrahlung photon energy was used. 7 different experimental setups and a total of 91 separate locations were prepared. Indium and cadmium-coated indium foils were irradiated at 10000 MU (Monitor Units - irradiation time) with $0^{\circ}, 90^{\circ}, 180^{\circ}$ and $270^{\circ}$ gantry angle (irradiation angle). In this regard, 
13 indium and then cadmium plated indium irradiations were made by placing in water used as a moderator, as shown in Figure 2.4. Water is a fine moderator which has a high neutron scattering cross section and thus allows thermalization [17].

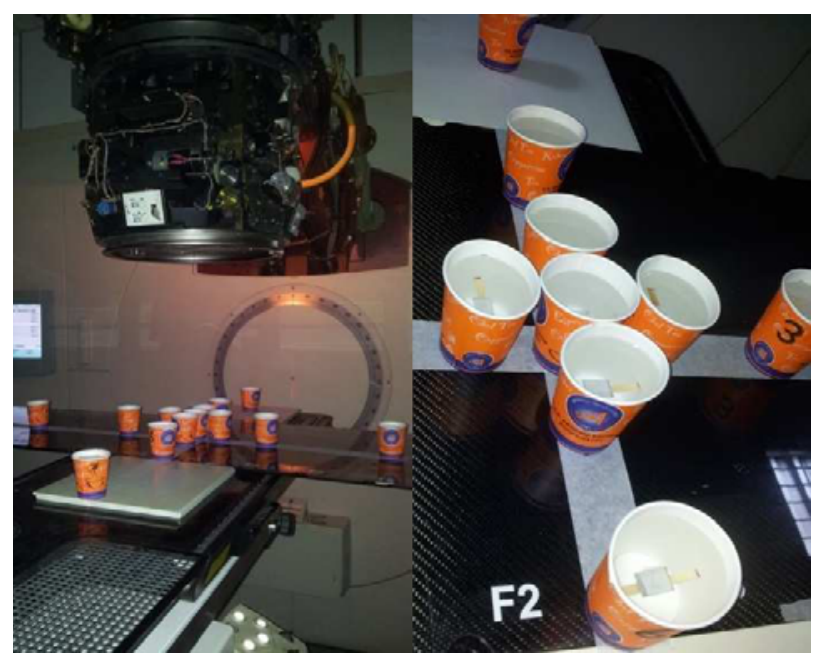

Figure 2.4. Flux measurement setup at the isocenter plane.

The cups that were used in the experiments have the same water height and the foils were placed at the same positions. Products of indium decay are shown in the diagram in Figure 2.5. ${ }^{116 \mathrm{~m}} \mathrm{In}\left(\mathrm{T}_{1 / 2}=54.2 \mathrm{~min}\right)$ with a $99.94 \%$ probability at $1293.4 \mathrm{keV}$ peak energy was counted and thus neutron flux information was obtained. The counting process was performed with the HPGe detector system and the data was analyzed by the GF3 gamma spectroscopy analysis software.

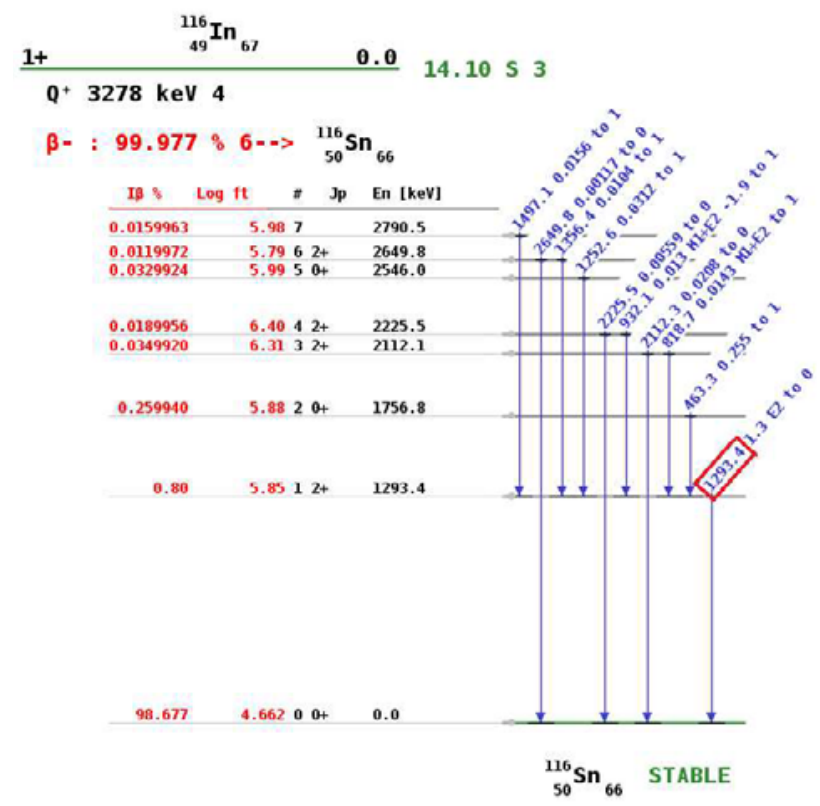

Figure 2.5. ${ }^{116}$ In Decay scheme [18]

Firstly, bare indium foils were irradiated and measurements were taken. The resulting count $\left(\mathrm{C}_{0}\right.$ (bare)) was recorded. Then indium foils coated with cadmium foils were irradiated, which are permeable to epithermal neutrons. Similarly, $\left(C_{0}(C d)\right)$ was measured and recorded. After the measurements, the thermal neutron flux was obtained using the following equations.

$$
\mathrm{C}_{0}=\frac{\mathrm{I} \lambda}{\varepsilon\left(1-\mathrm{e}^{-\lambda \mathrm{t}_{1}}\right)\left(\mathrm{e}^{-\lambda \mathrm{t}_{2}}-\mathrm{e}^{-\lambda \mathrm{t}_{3}}\right)}
$$

$\mathrm{C}_{0}$; irradiated foils saturation activity, $\mathrm{I}$; \# of counts between $t_{3}-t_{2}, \lambda$; radioactive decay constant of ${ }^{116}$ In foils $(\boldsymbol{\lambda}=0,0002130), \varepsilon$; the internal and geometric efficiency of the detector used in measurement, $\mathrm{t}_{1}$; foil irradiation time, $t_{2}$; remaining time between the start of the measurement and end of the irradiation, $t_{3}$; refers to the time period between end of the irradiation and end of the measurement.

$$
\begin{gathered}
\mathrm{C}_{0}(\text { bare })=\mathrm{N}\left(\phi_{\mathrm{th}} \sigma_{\mathrm{th}}+\phi_{\mathrm{r}} \sigma_{\mathrm{r}}\right) \\
\mathrm{C}_{0}(\mathrm{Cd})=\mathrm{N}\left(\phi_{\mathrm{r}} \sigma_{\mathrm{r}}\right)
\end{gathered}
$$

The terms used in the equations; $\mathrm{N}$; atom density of the foil $\left(\right.$ atoms $\left./ \mathrm{cm}^{3}\right), \phi_{\mathrm{th}}$; thermal neutron flux (neutron $\left./ \mathrm{cm}^{2} \cdot \mathrm{s}\right), \sigma_{\text {th }}$; thermal neutron absorption cross section of indium foils $\left(\mathrm{cm}^{2}\right), \phi_{\mathrm{r}}$; resonance neutron flux (neutron $\left./ \mathrm{cm}^{2} \cdot \mathrm{s}\right), \sigma_{\mathrm{r}}$ : resonance neutron absorption cross section of indium foils $\left(\mathrm{cm}^{2}\right)$.

The difference of counts taken by bare indium foils and indium foils coated by cadmium foils equals to the number of thermal neutrons.

$$
\mathrm{C}_{0}(\text { bare })-\mathrm{C}_{0}(\mathrm{Cd})=\mathrm{N} \phi_{\mathrm{th}} \sigma_{\mathrm{th}}
$$

To calculate the atom density of foils equation (2.6) was used.

$$
\mathrm{N}=\frac{\mathrm{m}}{\mathrm{M}} \mathrm{N}_{\mathrm{A}}
$$

To obtain thermal neutron flux, following procedures were performed.

$$
\begin{gathered}
\mathrm{R}=\frac{\mathrm{C}_{0}(\text { bare })}{\mathrm{C}_{0}(\mathrm{Cd})} \\
\mathrm{R}-1=\frac{\mathrm{N} \phi_{\mathrm{th}} \sigma_{\mathrm{th}}}{\mathrm{C}_{0}(\mathrm{Cd})} \\
\phi_{\mathrm{th}}=\frac{\mathrm{C}_{0}(\mathrm{Cd})}{\mathrm{N} \phi_{\mathrm{th}}}(\mathrm{R}-1)
\end{gathered}
$$

\section{Results}

Results of the analysis show that the maximum neutron flux in the room occurs at the top of the LINAC head towards the gun direction. This was expected since most of the neutrons are produced when the electron beam hits the tungsten target and at the primary 
collimator. Both the target and the primary collimator are located at the top of the gantry head. The maximum thermal neutron flux obtained was $1.2 \times 10^{5}$ neutrons $/ \mathrm{cm}^{2}$.s which is higher than a standard americium-beryllium (Am-Be) neutron source.

At the isocenter plane $(\mathrm{SSD}=100 \mathrm{~cm})$, the fluxes were $5.4 \times 10^{4}$ at the center, $1.5 \times 10^{4}$ at $2.5 \mathrm{~m}$ away and $9.9 \times 10^{3} \mathrm{n} / \mathrm{cm}^{2}$.s at the room wall which is $3.8 \mathrm{~m}$ away from isocenter. The flux at the maze entrance was measured nearly six in a ten thousand less $\left(81 \mathrm{n} / \mathrm{cm}^{2} . \mathrm{s}\right)$.

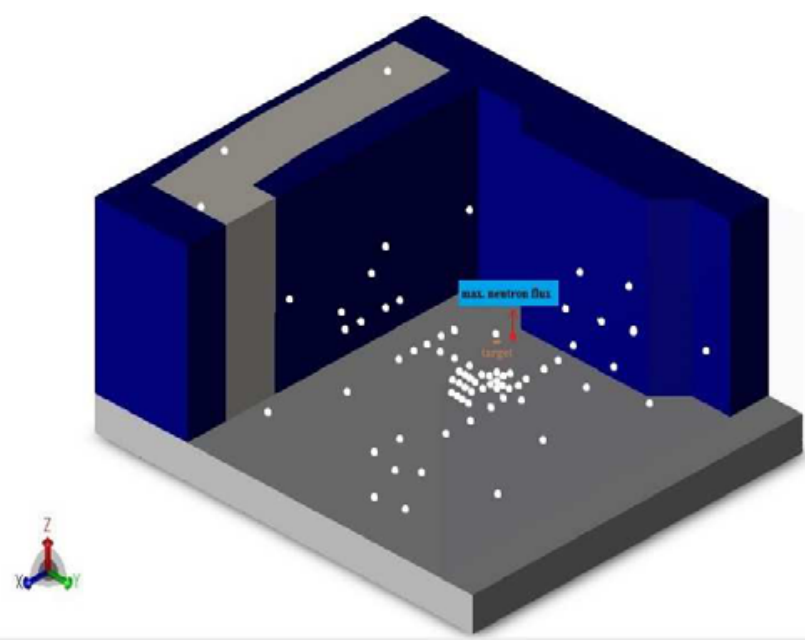

Figure 3.1. Positions of measurement (91 points) in the linear accelerator room

To set up an example, thermal neutron flux distributions are shown for 2 experimental setups of the total of 7 experimental setups.

\begin{tabular}{|l|}
\hline Experimental Parameters \\
X-Ray \\
Gantry $=0^{0}$ \\
$10000 \mathrm{MU}$ (avg. irradiation time $30 \mathrm{~min})$ \\
$18 \mathrm{MV}(18 \mathrm{MeV}$ brems spectrum) \\
$40 \mathrm{x} 40 \mathrm{~cm}^{2}$ area (collimation) \\
$\mathrm{SSD}=100 \mathrm{~cm}$ (Distance to target) \\
\hline
\end{tabular}

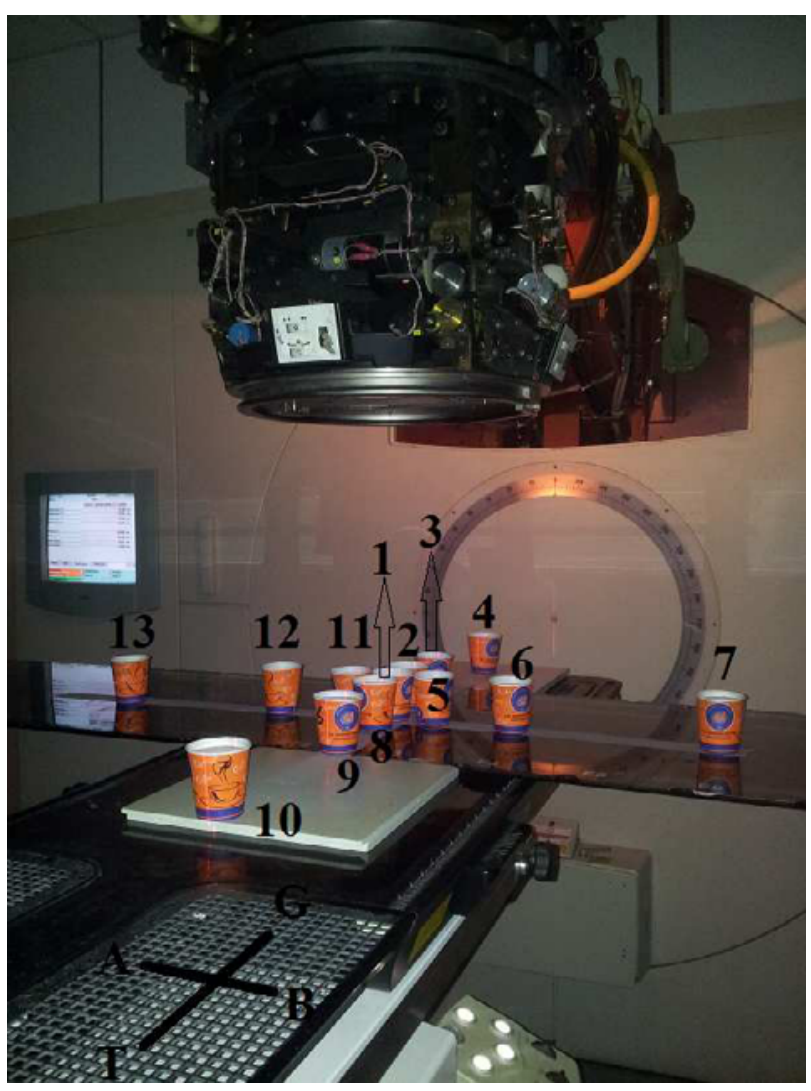

Figure 3.2. Location of indium and cadmium foils for experiment \#1

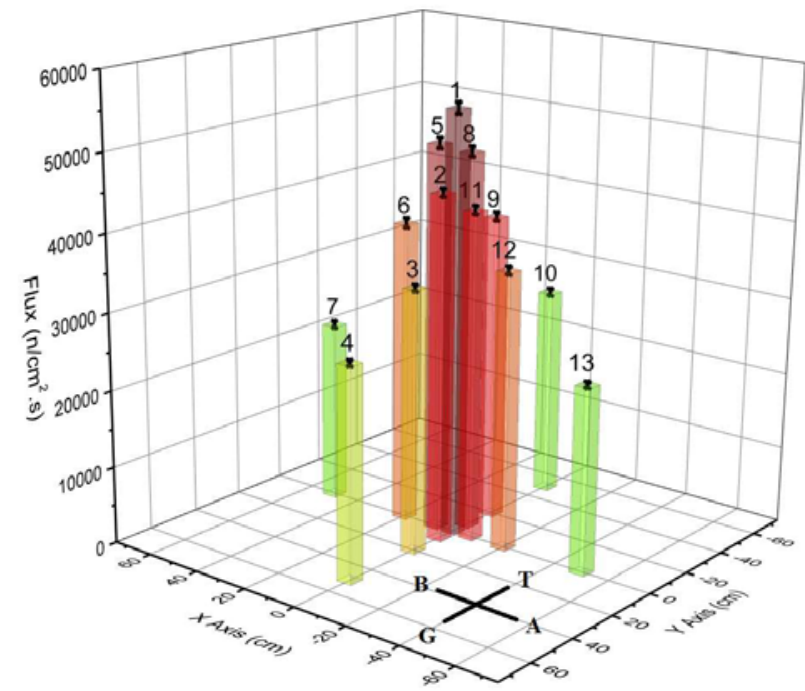

Figure 3.3. Space dependent photoneutron flux distribution graph obtained from experiment \#1 


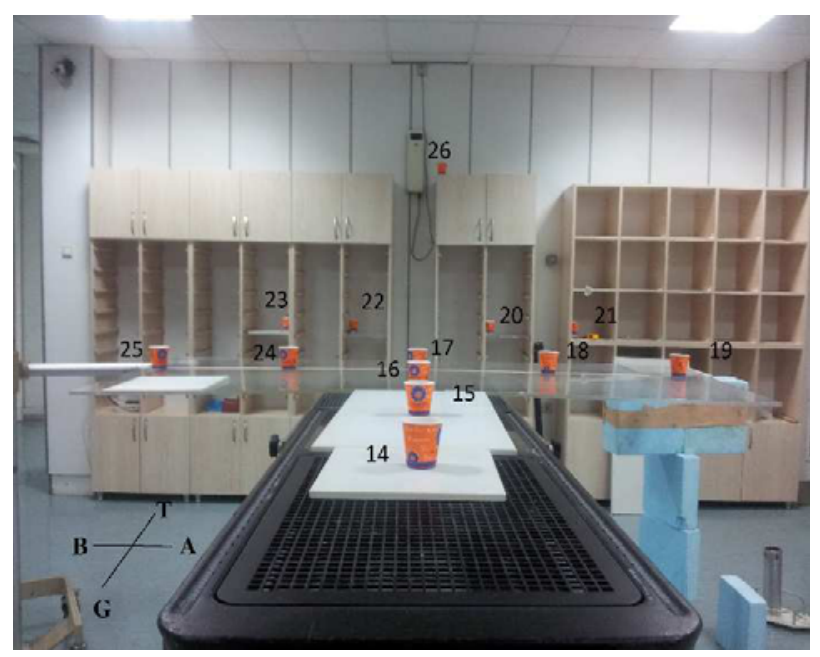

Figure 3.4. Location of indium and cadmium foils for experiment \#2

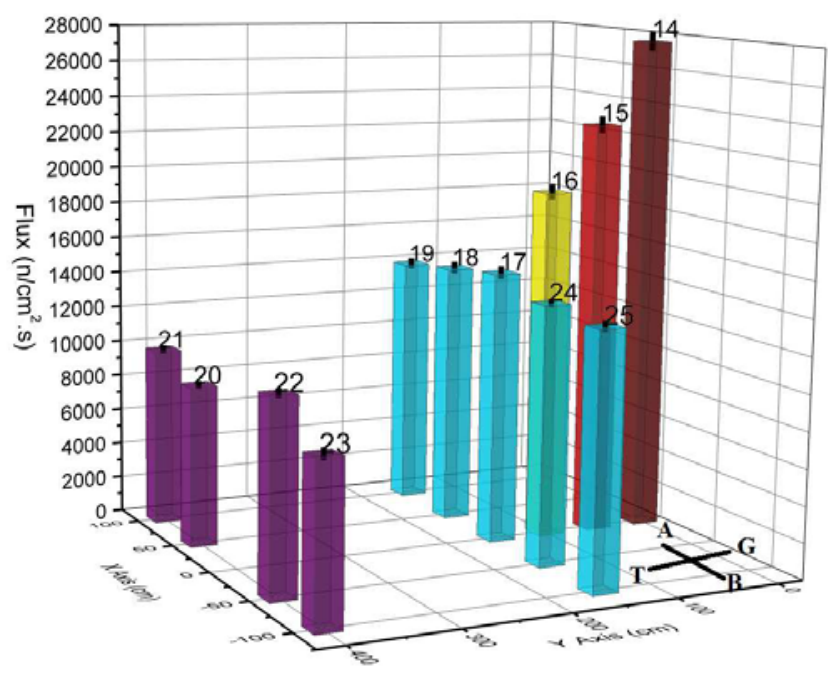

Figure 3.5. Space dependent photoneutron flux distribution graph obtained from experiment \#2

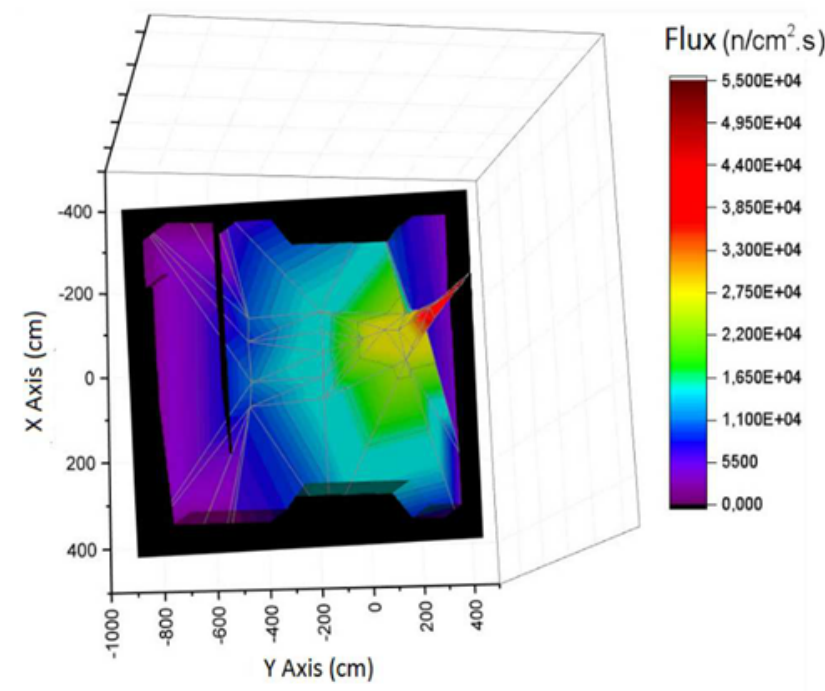

Figure 3.6. Thermal neutron flux distrubution in LINAC room $(\mathrm{SSD}=100 \mathrm{~cm}, 10000 \mathrm{MU})$

\section{Discussion}

When obtained results were analyzed, maximum photoneutron flux occurred at the center of the LINAC and showed a descending gaussian distribution to the walls. Thermal photoneutron flux at the field center was $1.2 \times 10^{5} \pm 740 \mathrm{n} / \mathrm{cm}^{2}$.s. At the doorway just after the door, thermal neutron flux was $81 \pm 46 \mathrm{n} / \mathrm{cm}^{2}$.s.

A similar study was performed by Yücel et al. A Varian Clinac DHX device and RW3 solid phantom were used. For the NAA, gold and cadmium-coated gold foils were used. The measurements were performed at the field center, field edges and different positions in the room at depths of 0,5 and $10 \mathrm{~cm}$ water. During the experiment; gantry angle was zero, irradiation time was $5000 \mathrm{MU}$, the field size was $20 \times 20 \mathrm{~cm}^{2}$ and SSD was $100 \mathrm{~cm}$. Thermal neutron flux measurement results were at surface $(\mathrm{d}=0 \mathrm{~cm}) 6.65 \pm 0.20 \times 10^{5} \mathrm{n} / \mathrm{cm}^{2} . \mathrm{s}$, at depth 5 $\mathrm{cm} 3.73 \pm 0.11 \times 10^{6} \mathrm{n} / \mathrm{cm}^{2}$.s and depth $10 \mathrm{~cm}$ of water $2.18 \pm 0.06 \times 10^{6} \mathrm{n} / \mathrm{cm}^{2}$.s. At the door, flux was $2.36 \pm 1.30 \times 10^{2} \mathrm{n} / \mathrm{cm}^{2} . \mathrm{s}$ [19]. These results are comparable with our findings. The differences occur from different design-brand of the accelerator and other measurement parameters.

Esposito et al, studied NAA with Elekta Precise linear accelerator device which is nearly the same device that we used in the experiment. The photon energy was chosen as $18 \mathrm{MV}$. The field size was $15 \times 15 \mathrm{~cm}^{2}$ and gold foils were used for NAA. After the measurement, the maximum neutron flux was observed at $1 \mathrm{~cm}$ away from the center and at SSD $100 \mathrm{~cm}$ flux was $48 \%$ of the maximum flux. At the maze entry, the flux was $15 \%$ of the maximum flux [20]. The results were given in terms of dose values so it would be appropriate to compare the results relatively with our study. When the results were compared with respect to the maximum flux, they appeared to be quite similar. In our study, $100 \mathrm{~cm}$ away from the center, the flux equals to $45 \%$ of the maximum neutron flux, and at $1.5 \mathrm{~m}$ away from center it is $42 \%$ of the maximum flux. At the maze entry it was $13 \%$ of maximum flux.

Konefal et al, studied with a 15 MV Siemens Primus device. They used indium and cadmium-coated indium foils for the NAA. They took measurements at various points in treatment room. As a result, the maximum thermal neutron flux was obtained at the center of field at SSD $100 \mathrm{~cm}$. Thermal neutron flux at the wall was $67 \%$ of the measurement taken at the field center. $1.5 \mathrm{~m}$ away from the center, the measurement showed that the thermal neutron flux was $91 \%$ of the maximum thermal neutron flux. The measurement that was taken at the intersection of the maze was $21 \%$ of the maximum thermal neutron flux [14]. In our study, the thermal neutron flux values were $33 \%, 42 \%$, and $13 \%$ respectively.

Liu et al, used a Varian Clinac 21EX linear accelerator. Photon energy was chosen as $15 \mathrm{MV}$ and indium foils were used in this study. The thermal neutron 
flux change was investigated from field center to the field edge. The results showed that the thermal flux ratio to the maximum flux at $20 \mathrm{~cm}$ away from center was $80 \%$ for direction A (Left side of the LINAC), and for direction B (right side) at same distance the result was $50 \%$ of the maximum thermal neutron flux [21]. The thermal neutron fluxes at the same points in our study were $65 \%$ and $71 \%$ respectively. One should expect similar results due to symmetry.

Sanchez et al, used indium foils in the linear accelerator room during their experiment. The thermal neutron flux at SSD $100 \mathrm{~cm}$ and field size of $30 \times 30 \mathrm{~cm}^{2}$ was $7.28 \times 10^{4} \mathrm{n} / \mathrm{cm}^{2} . \mathrm{s}$ [22]. In our study the thermal neutron flux at same condition was obtained as $5.4 \times 10^{4} \pm 744 \mathrm{n} / \mathrm{cm}^{2}$.s. The order of results is the same for the two studies. However, the model of linear accelerator was not specified.

In this study, we used indium and cadmium foils for the measurement of the thermal neutron flux at various locations throughout the room. The maximum thermal neutron flux was detected just above the head of the LINAC towards the gun direction $\left(\mathrm{G}=0^{0}\right)$. The neutron flux decreased from the field center towards the edge of the room which followed a gaussian distribution. The studies that were compared with our study included some different parameters which caused some deviations in the results. Different parameters were; different types of activation foils, amount of given MU, model and brand of LINACs and types of detectors. Nedaei et al, showed that the Varian brand devices caused three times more photoneutron than the Elekta brand devices [23]. Liu et al, discussed that the experiments that were carried out with activation foils caused a standard deviation between $12.2 \%-13.9 \%$ because of normalization of foils and the self-absorption phenomena [21].

After an extensive literature research, we can say that measurement of thermal neutron flux at 91 different locations inside a radiotherapy room was the most comprehensive study that was carried out up to date. The experimental parameters varied extensively; such as, different gantry angles, different MU values, inside and outside field measurements, etc. Thus, we could map the thermal neutron flux distribution throughout the room.

\section{Conclusions}

Recently, radiotherapy in the treatment of cancer patients has become an important modality. The radiation energy used is increasing with the development of new technologies. But if the necessary measures are not taken, increasing the radiation energy can give damage to patients, employees and environment. One should understand the consequences of photoneutron production in linear accelerators and should analyze the flux and energy of neutrons which were formed in the treatment rooms.
In our study, the neutron flux distribution within the bunker was measured with detail using 91 points. The flux decreases as we move away from the isocenter, which is compatible with the literature. The magnitude of the neutron fluxes shows that there is a significant amount of neutron dose within the room. The corresponding neutron dose to the patient however is only $0.1-0.3 \%$ of the total dose. However, neutrons have a high RBE and this unwanted dose is not calculated with the treatment planning system (TPS).

Photoneutron production is an interesting research topic which has both experimental and simulation legs. The future work would be to compare the results with the Monte Carlo simulations.

\section{References}

1. A. Jemal, T. Murray, A. Samuels, A. Ghafoor, E. Ward, M.J. Thun, Cancer Statistics. American Cancer Society. 53: 5-26 (2003)

2. J.I. Spicka, H.S. Kim, D.W. Oh, V. Marable, K. Fleury, Equal surface dose compensation. American Association of Medical Dosimetrists. 14: 287-290 (1989)

3. F. Biltekin, M. Yeğiner, G. Özyiğit, Investigating infield and out-of-field neutron contamination in highenergy medical linear accelerators based on the treatment factors of field size, depth, beam modifiers, and beam type. Physica Medica. 31: 517-523 (2015)

4. V. Vylet, J.C. Liu, Radiation Protection at High Energy Electron Accelerators. Oxford Journals., 96: 333-343 (2011)

5. B. Juste, S. Morató, R. Miró, G. Verdú, S. Díez, MCNP6 unstructured mesh application to estimate the photoneutron distribution and induced activity inside a linac bunker. Radiation Physics and Chemistry. (2016)

6. International Commission on Radiological Protection. ICRP Publication 60, Recommendations of the International Commission on Radiological Protection (1990)

(http://www.icrp.org/publication.asp?id=icrp\%20pub lication\%2060)

7. R.M. Howell, M.S. Ferenci, Investigation of secondary neutron dose for $18 \mathrm{MV}$ dynamic MLC IMRT delivery. American Association of Physicists in Medicine 32: 786-793 (2005)

8. J.P. Lin, W.C. Liu, C.C. Lin CC, Investigation of photoneutron dose equivalent from high-energy photons in radiotherapy. Applied Radiation and Isotopes, 65: 599-604 (2007)

9. L. Sajó-Bohus, H.R. Vega-Carrillo, H.S. Virk, SSNTD technique in photo-neutron applications. Solid State Phenomena. 239: 180-214 (2015)

10. E.D. Conrad, Transient Radiation Effects In Electronics. Advances in Nuclear Science and Technology. 3: 143-203 (1966)

11. A. Naseria, A. Mesbahia, A review on photoneutrons characteristics in radiation therapy with high-energy 
photon beams. Reports of practical oncology and radiotherapy. 15: 138-144 (2010)

12. M. Králik, K. Turek, Characterisation of neutron fields around high energy X-ray radiotherapy machines. Rad. Prot. Dosim. 110: 503-507 (2004)

13. C.L. Allred, Effects of Radition On Silicon and Borosilicate Glass. MIT Materials Science and Engineering, Massachusetts (2003)

14. A. Konefał, A. Orlefb, M. Dybekc, Z. Maniakowskib, K. Polaczek-Grelika, W. Zippera, Correlation between radioactivity induced inside the treatment room and the undesirable thermal/resonance neutron radiation produced by linac. Physica Medica. 24: 212-218 (2008)

15. International Nuclear Data Committee, IAEA Annual Report, Atlas of Neutron Capture Cross Sections (1997) (http://www.iaea.org/inis/collection/NCLCollectionS tore/ Public/28/060/28060364.pdf)

16. J.L. Lawson, J.M. Cork, The radioactive isotope indium. Physical Review. 52: 531-535 (1937)

17. J.R. Lamarsh, A.J. Baratta, Radiation Shielding. In: Horton MJ ed. Introduction to Nuclear Engineering. 3rd ed. New Jersey: Prentice Hall p: 548-604 (2001)

18. International Atomic Energy Agency (IAEA), Nuclear Data Services, ${ }^{116}$ In Decay Radiation Scheme (https://wwwnds.iaea.org/relnsd/vcharthtml/VChartHTML.html)

19. H. Yücel, İ. Çobanbaş, A. Kolbaşı, A.Ö. Yüksel, V. Kaya, Measurement of Photo-Neutron Dose from an 18-MV Medical Linac Using a Foil Activation Method in View of Radiation Protection of Patients. Nuclear Engineering and Technology. 48: 525-532 (2016)

20. A. Esposito R. Bedogni, L. Lembo, M. Morelli, Determination of the neutron spectra around an 18MVmedical LINAC with a passive Bonner sphere spectrometer based on gold foils and TLD pairs, Radiation Measurements. 43: 1038-1043 (2008)

21. M. Liu, S.S. Huang, W.S. ShanLiu, D.M. Yea, L.K. Pan LK, C.Y. Chen CY, Distribution of spatial photo neutrons inside a $70 \mathrm{~kg}$ water phantom via neutron activation analysis. Applied Radiation and Isotopes. 68 : 1816-1821 (2010)

22. F. Sanchez, G. Madurga, R. Arrans, Neutron measurements around an $18 \mathrm{MV}$ linac. Radiotherapy and Oncology. 15: 259-265 (1989)

23. H.A. Nedaie, H. Darestani, N. Banaee, N. Shagholi, K. Mohammadi, A. Shahvar, E. Bayat, Neutron dose measurements of Varian and Elekta linacs by TLD600 and TLD700 dosimeters and comparison with MCNP calculations. Journal of Medical Physics. 39: 10-17 (2014) 